\title{
Paratesticular Malignant Mesothelioma
}

National Cancer Institute

\section{Source}

National Cancer Institute. Paratesticular Malignant Mesothelioma. NCI Thesaurus. Code C162477.

A rare malignant mesothelioma that arises from the tunica vaginalis. 